\title{
Proposta de arquitetura baseada em agentes inteligentes integrados em ambientes e-learning
}

\author{
Proposal for architecture based on intelligent agents integrate in e- \\ learning enviroments
}

\section{Propuesta de arquitectura basada en agentes inteligentes integrados en entornos de e-learning}

Francielli Freitas Moroㄹ; Liane Margarida Rockenbach Tarouco²; Rosa Maria Vicari³

\section{RESUMO}

Encontrar soluções para aproximar professor e aluno em ambientes e-learning como o Moodle e diminuir os índices de evasão nos cursos é um desafio enorme e potencializado com a pandemia de SARS-COV-2. Para isso, torna-se necessário encontrar soluções inteligentes que forneçam reflexão, interação, motivação, acompanhem o processo de aprendizagem do aluno e apoiem o professor. O presente artigo apresenta uma proposta de arquitetura de ambiente e-learning baseado em agentes inteligentes, para minorar problemas do ensino de disciplinas na área de matemática nas engenharias. Para isso, foi realizada uma pesquisa na literatura, apontado os principais relacionados a e-learning e a matemática na engenharia, e possíveis soluções baseadas em agentes inteligentes como chatbots e nas arquiteturas de Sistemas Tutores Inteligentes. Além da proposta, foi realizada uma breve discussão de como a arquitetura ajudaria a resolver os problemas levantados e de como aplicá-la.

Palavras-chave: Matemática; Engenharia; E-learning; Sistemas Tutores Inteligentes; Chatbots.

\begin{abstract}
Find solutions to bring teacher and student closer together in e-learning environments such as Moodle, and decreasing dropout rates in courses is a huge and potential challenge with the SARS-VOC-2 pandemic. For this, it is necessary to find intelligent solutions that provide reflection, interaction, motivation, follow the student's learning process and support the teacher. This article presents a proposal for an e-learning environment architecture based on intelligent agents, to alleviate problems in the teaching of engineering mathematics subjects. For this, a literature search was carried out, pointing out the main ones related to e-learning and mathematics in engineering, and possible solutions based on intelligent agents such as chatbots and on the architectures of Intelligent Tutoring Systems. In addition to the proposal, there was a brief discussion of how architecture would help to solve the problems raised and how to apply it.
\end{abstract}

Keywords: Math; Enginnering; E-learning; Intelligent Tutoring System; Chatbots.

\footnotetext{
${ }^{1}$ Doutoranda no Programa de Pós Graduação em Informática na Educação na Universidade Federal do Rio Grande do Sul (UFRGS), Mestre no Programa de Pós Graduação em Tecnologias da Informação e Comunicação (PPGTIC) pela Universidade Federal de Santa Catarina (UFSC). Bacharel em Tecnologias da Informação e Comunicação (TIC) também pela UFSC. E-mail: franf_m@hotmail.com

2 Professora titular da Universidade Federal do Rio Grande do Sul (UFRGS) e coordenadora do Programa de Pós-Graduação em Informática na Educação. Graduada em Licenciatura em Física, mestrado em Ciências da Computação e doutorado em Engenharia Elétrica/Sistema Digitais. E-mail: liane@penta.ufrgs.br

${ }^{3}$ Professora titular da Universidade Federal do Rio Grande do Sul (UFGRS) no Instituto de Informática (INF) e coordenadora da Cátedra UNESCO em Tecnologias de Comunicação e Informação na Educação. Possui pós doutorado em Cognitive Science, doutorado em Engenharia Electrotécnica e Computadores, mestrado em Ciências da Computação e graduação em Tecnólogo em Processamento de Dados. E-mail: rosa@inf.ufrgs.br
} 


\section{RESUMEN}

Encontrar soluciones para acercar al profesor y al alumno en entornos de e-learning como Moodle y reducir las tasas de deserción en los cursos es un desafío enorme y potencial con la pandemia del SARS-VOC-2. Para ello, es necesario encontrar soluciones inteligentes que aporten reflexión, interacción, motivación, sigan el proceso de aprendizaje del alumno y apoyen al docente. Este artículo presenta una propuesta de arquitectura de entorno de e-learning basada en agentes inteligentes, para paliar problemas en la enseñanza de las asignaturas de ingeniería matemática. Para ello, se realizó una búsqueda bibliográfica, señalando los principales relacionados con el e-learning y las matemáticas en la ingeniería, y posibles soluciones basadas en agentes inteligentes como los chatbots y en las arquitecturas de los Sistemas de Tutoría Inteligente. Además de la propuesta, hubo una breve discusión de cómo la arquitectura ayudaría a resolver los problemas planteados y cómo aplicarla.

Palabras clave: Matemáticas; Ingenieria; Aprendizaje electrónico; Sistemas de Tutoría Inteligente; Chatbots.

\section{INTRODUÇÃO}

O e-learning ${ }^{1}$ desempenha um potencial papel na educação, ainda mais intensificado com a pandemia de SARS-COV-2 e por isso torna-se necessário investigar as melhores formas de explorar suas vantagens. Vários teóricos educacionais têm examinado extensivamente as variáveis motivacionais, revelando que as motivações intrínsecas e extrínsecas dos estudantes têm influenciado nos resultados da aprendizagem (Hesegawa, Sakuta e Ugurlo, 2014). Além disso, diversas outras variáveis como reflexão, comportamento, engajamento, eficácia, avaliação, preparação e envolvimento com metodologias de aprendizagem vem sendo estudadas por diversos pesquisadores a fim de promover uma educação mais inclusiva, personalizada e flexível.

Esses pontos se tornam cada vez mais difíceis de medir em ambientes e-learning, com as aplicações já atribuídas, trazendo dificuldades aos professores de acompanhar o aprendizado da sua turma de forma real e potencial, principalmente nas áreas de Matemática (Akugizibwe e Ahn, 2019).

Um agente inteligente pode ser aplicado para resolver questões em diversas áreas e subáreas e aplicar esse tipo de entidade, na engenharia, por exemplo, pode reduzir erros exponenciais, potencializar aprendizagens, e permite o acompanhamento mais aproximado do aluno ao ambiente e os materiais disponibilizados. Um agente é definido como um sistema encapsulado situado em um determinado ambiente capaz de agir de forma flexível e autônoma e pode se comunicar e cooperar com outros agentes no mesmo ambiente, possuindo também algumas características como: autonomia, habilidade social, reatividade e pró-ativismo (Russel e Norvig, 2004).

O objetivo deste artigo é apresentar pesquisas sobre o uso de agentes inteligentes em ambientes como Sistemas Tutores Inteligentes (STI) e Ambientes Virtuais de Aprendizagem (AVA), para apoiar o ensino de matemática na engenharia e propor uma arquitetura baseada na literatura e na atribuição de agentes conversacionais, que promova a diminuição de problemas encontrados em ambientes $e$ learning em cursos da área, buscando evitar evasão.

O presente estudo foi dividido da seguinte forma a partir desta introdução. Na seção 2 são apresentados os problemas principais nas áreas das disciplinas que envolvem matemática na engenharia. Na seção 3, são apresentados a relação de agentes inteligentes na educação e alguns estudos referentes às aplicações destes ambientes nas áreas. Na seção 4, são apresentados estudos referentes a arquitetura de ambientes e-learning que utilizam agentes inteligentes para personalizar a educação. Na seção 5, é apresentada a arquitetura proposta baseada nas possíveis soluções 
encontradas na literatura, e discussões pertinentes, em que por fim na seção 6 são alocadas as contribuições destes trabalhos, conclusão e referencias.

\section{CONTEXTUALIZAÇÃo}

Após serem realizadas oficinas de metodologias ativas com alguns professores de graduação da Universidade Federal do Rio Grande do Sul (UFRGS) que atuam nas engenharias e utilizam a plataforma MOODLE como ambiente e-learning para apoio a aprendizagem, foram relatadas algumas dificuldades relacionadas à utilização de ambientes virtuais em suas matérias. Entre elas estavam a falta de subsunçores por parte dos alunos, a falta de colaboração, o senso de reflexão sobre as atividades propostas ou vídeos disponibilizados, a falta de direcionamento do sistema para que o aluno entenda quais seus pontos fracos e possa reforçá-los, a falta de acompanhamento do professor no horário que o aluno está fazendo uma determinada atividade, e a evasão por falta dessa atenção e da dificuldade proveniente das matérias que envolvem cálculos matemáticos.

Radovic, Maric e Passey (2018) destacam alguns pontos em que as tecnologias podem fornecer benefícios para o ensino de matemática, se bem planejadas, como: fornecimento rápido e confiável de feedback, criação de exemplos reais ao explorar problemas de matemática; maiores conexões entre representações e mudanças em objetos matemáticos; trabalhos com imagens dinâmicas e representativas.

Loch e Lamborn (2015) mostram em sua pesquisa o quanto matemática é elemento fundamental na educação de engenharia e, ao qual o aluno tem uma responsabilidade enorme quanto a sua própria aprendizagem nesse sentido, mesmo que ele não considere ou goste das disciplinas. Por isso, é necessário que o ambiente e-learning se adapte a sua forma de pensar, oferecendo subsídios para diminuir dificuldades, ilustrar problemas e oferecer acompanhamento.

\section{AGENTES INTELIGENTES NO E-LEARNING}

Um ambiente e-learning pode possuir diversos agentes para acompanhar a trajetória dos estudantes, sugerir materiais, gerenciar a aprendizagem, coletar informações, prevenir erros e realizar diversas outras funções. Segundo Herpich et al (2014), Agentes inteligentes devem perceber o ambiente no qual atuam, o que ocorre através de sensores, para assim adotar as ações possíveis para cada situação. Esses agentes são usados na construção de sistemas tutores, principalmente voltados a educação. Frozza et al (2011) destaca que utilizar sistemas com agentes para ensino e aprendizagem é algo que vem ganhando cada vez mais ênfase, e desenvolvimento por instituições, diante as facilidades e tomadas de decisão que realizam. Em resumo, Santos et al (2018) concluem que um agente dentro de um STI, por exemplo, atua como monitores, observando as ações dos alunos e os ajudam durante o seu aprendizado e enquanto um pode armazenar dados de diversidade entre os usuários, respeitando o nível de aprendizagem individual, outro pode armazenar estratégias pedagógicas para auxiliar um aluno em determinado domínio, trocando informações entre si para encontrar melhores formas de adaptação.

Leonhardt (2005) frisa que é necessário que se conheça o ambiente de atuação do agente e verifiquese quais os objetivos específicos o agente deve atender, para que se consiga traçar ações e designar quais percepções deve-se obter do ambiente. 
Aplicações como agentes conversacionais podem ser atribuídas, em colaboração com os agentes do sistema e atuar diretamente de forma perceptiva com o aluno, podendo oferecer uma maior proximidade a partir da interação, sugerindo materiais de apoio ou que supram as deficiências de prérequisitos, como um tutor humano.

Alexandru e Tirziu (2015) frisam que com a interação, os estudantes são engajados em resolver problemas reais e o conhecimento existente é ativado como base a ter novos conhecimentos, integrando o novo conhecimento adquirido as coisas do dia-a-dia.

Dinggil, Oh e Rice (2017) destacam que poucos sistemas são usados concretamente em ferramentas de educação online, e eles nem sempre são efetivos, e falham para objetivos instrucionais. Além disso eles promovem uma aprendizagem passiva e simples na apresentação de materiais instrucionais. Como a interação com agentes ocorre de maneira efetiva, eles podem ser relevantes na educação online, se bem utilizados, e podem promover benefícios como a persistência do aluno no curso a partir de experiências construtivas. Requer-se para isso conteúdo relacionado, instantâneo e qualidade de feedback para instruir o aluno ou os pares.

\subsubsection{Agentes Conversacionais}

Agentes conversacionais, popularmente conhecidos como chatbots, são aplicações computacionais que ao interagir diretamente com o humano conseguem manter uma conversa de forma sequencial ou próxima a um diálogo real (Io e Lee, 2017).

Existem alguns agentes conversacionais educacionais modelados com Artificial Intelligence Markup Language (AIML) ${ }^{2}$, e baseados no chatbot ALICE, como o Elektra e a Metis. Além disso, existem também chatbots modelados por ontologias, regras, e outras técnicas de IA. Os chatbots desenvolvidos atualmente a partir de modelo para empresa, buscam solucionar problemas de lembrança e cognição, deixando a conversa mais natural. Para isso, algumas empresas como GOOGLE e IBM através do Watson Assistant, oferecem serviços de chatbot estruturados além da base alocada, com a utilização de classificadores e dicionários de Linguagem Natural de Máquina (NLP) e Redes Neurais Artificiais $^{3}$ (Moro, 2019). Kuyven et al (2018) apresenta em seu trabalho uma revisão sistemática que apresenta diversas pesquisas brasileiras sobre agentes conversacionais na educação, como a pesquisa de Paschoal, Chicon e Falkemback (2017) que apresenta o Ubibot um chatbot integrado ao moodle que objetiva prover suporte ao ensino na disciplina de Engenharia de Software.

O estudo de Donggil, Oh e Rice (2017) Apresentou o design e desenvolvimento de um chatbot que promove a interação sistema-aprendiz em tarefas de reflexão sobre as atividades propostas no $e$ learning. O agente criado perguntava ao aluno questões como "Que conhecimento relevante você adquiriu sobre determinada tarefa?" e à medida que o usuário o agente indagava sobre outras questões para que o aluno permaneça ativo durante o aprendizado. Um acompanhamento mais próximo do aluno a partir de um chatbot pode diminuir fatores como evasão nos cursos à distância.

\subsubsection{E-learning nas áreas de Matemática e Engenharia}

Ferreira Filho (2008) apresenta um Sistema Tutor Inteligente para ensino de problemas de Engenharia para Engenharia Civil utilizando a abordagem de $\mathrm{PBL}^{4}$. Ao escolher um problema no STI o aluno é levado a área de resolução deste problema em específico em que encontra o enunciado e 
uma simulação de uma situação real ocorrente na prática profissional para propor uma solução do problema.

A pesquisa de Frigo (2002) apresenta um ambiente interativo multiagente baseado no sistema MATHEMA que é inspirado na abordagem de construtivismo de Vygotsky e implementações de agente SATA $^{5}$ para auxiliar os alunos de Engenharia de controle e Automação industrial a resolverem problemas na disciplina de fundamentos de estrutura de Informação.

Jacques et al (2013) apresentam Path2MATH, um Sistema Tutor Inteligente baseado nos modelos clássicos para auxiliar no ensino e acompanhar o aluno na construção de conhecimento sobre Álgebra Linear. Para a mesma área, Crown et al (2010) apresenta um agente conversacional chamado Anne G. Neering, desenvolvido com AIML, para envolver o aluno através da motivação e foco nos interesses, objetivos e aspirações do aluno. O Chatbot interativo ajudou a estabelecer competência de conteúdo e envolvimento pessoal, tratando as perguntas dos alunos como uma fonte de problemas para verificar e investigar. De acordo com os autores, notou-se que a ferramenta força os alunos a relacionar os problemas com os pré-requisitos cognitivos.

A pesquisa de Aguiar, Tarouco e Reategui (2014) apresenta o agente conversacional chamado BLAZE que foi desenvolvido utilizando uma estrutura AIML com uma base de respostas proporcionada por alunos superdotados e visava apoiar alunos com dificuldade a resolver problemas matemáticos. Esse agente atuou como um assistente treinado e confiável e foi capaz de interagir com alunos mostrando estratégias para resolução de novos problemas a partir da aprendizagem significativa. Obteve-se um resultado positivo em relação as notas dos alunos, que eram mais altas com a ajuda do agente.

\section{EXEMPLOS DE ARQUITETURAS PROPOSTAS NA LITERATURA}

A arquitetura proposta por Ferreira Filho (2008) utiliza 3 módulos principais: a) o modulo de domínio que possuí um acervo de recursos educacionais para Engenharia de Fundações disponibilizado em pastas na arquitetura de aplicação em que são descritos e mapeados a partir de metadados estruturados em um Banco de Dados; b)o módulo do aluno responsável pela abstração do conhecimento do aluno baseado na técnica overlay e; c)um modelo do tutor que propõe um conjunto de estratégias, baseado em regras de um sistema especialista. Os problemas são alocados de acordo com o grau de dificuldade a partir das respostas dos alunos.

O Sistema apresenta os problemas que compõe o nível, o aluno escolhe um e pode ou não buscar mais informações a respeito dele. A qualquer momento o aluno pode armazenar informações provenientes do repositório educacional ou de fontes e a partir daí o aluno determina os parâmetros para resolução dos problemas, e atribui valor a eles para selecionar assim a resposta adequada. 0 objetivo principal do sistema, é fornecer ao aluno informações importantes de acordo com sua dificuldade e com requisitos, para que ele possa aprender com exemplos reais de um Engenheiro.

Ainda fica em aberto, como perspectiva, a integração de uma ferramenta que forneça feedback imediato sempre que os alunos desejarem fornecendo dicas, sugestões e questionamentos, ao qual o papel de um chatbotseria extremamente adequado para atuar com maior proximidade com o aluno.

Alexandru e Tirziu (2015) se baseiam na arquitetura tradicional a partir de 5 módulos: o módulo tutor que tem funções didáticos-pedagógica e classifica para tomar uma decisão de acordo com cada 
perfil, e coleta informações sobre as atividades e recursos propostos; o módulo base de conhecimento, que coleta dados pessoais de interação e do ambiente de desenho instrucional; o módulo do estudante, que possuí agentes inteligentes para modelagem do estudante e cria os modelos de aluno de acordo com sua navegação no ambiente, nível de conhecimento, cooperação e aprendizagem além de prover a atualização de informação, monitorando os módulos e modificando se houver necessidade; o módulo de interface, que determinada determina o tipo mais apropriado tipo de interface para cada usuário baseado em software e hardware; e um módulo de tomada de decisão instrucional, que decide a estratégia de aprendizagem e descreve a função como criada. Ele pega os modelos de usuário criado e envia conteúdo de acordo com o estilo de aprendizagem.

Palomino (2013) apresentou um modelo com agentes inteligentes para o Moodle baseado na arquitetura de um Sistema Tutor Inteligente, buscando permitir adaptabilidade, proximidade e personalização. Foi estudada a base de dados do Moodle, estendida através da criação de novas tabelas e modificou-se o código de instalação do AVA, ao qual foram implementados agentes, através do envio de mensagens dos alunos e da configuração dos recursos e atividades a serem disponibilizadas nas disciplinas pelos professores de forma individual e adaptativa para cada aluno. No modelo proposto o professor escolhe o modelo pedagógico a ser usado, o material didático (leitura, artigo, vídeos...) e atividades a serem realizadas que ele considera necessário para explorar da melhor forma todos os recursos. Ele configura um agente denominado BEDEL responsável por ajudá-lo como se fosse um professor auxiliar. Há também um agente TUTOR que orienta o aluno indicando mudanças no desempenho dele, cada vez que uma nova atividade é avaliada, e o incentiva a melhorar determinado conteúdo quando este tiver uma queda no seu desempenho. Esse sistema foi implementado baseado em um interpretador JASON ${ }^{6}$ lógico, ao qual a intenção do usuário é valorizada e nos artefatos SQL.

A arquitetura proposta por Ghouch, EM-Naimi e Kouissi (2015) é interessante pois atribuí uma maior quantidade de agentes. A proposta é um sistema de aprendizagem multiagente adaptativo baseado em Incremental hybrid case-based reasoning (IHCBR) que é formado por interações passadas de cada estudante e no modelo de estilo de aprendizagem de Felder e Silverman (FSLSM) constituído por 4 características, sendo elas: percepção, entrada, processamento e entendimento. Através desta junção é possível criar os caminhos de aprendizagem de maneira personalizada e dinâmica e prever comportamentos futuros. Na arquitetura proposta há: agente interface, que é responsável pela interação humano-máquina, pela conexão e registro de novos usuários e pela comunicação e criação de agente mobile. Um agente guia, que apresenta e exibe os cursos adaptados a cada perfil e os adapta de acordo com o cálculo de correspondência entre o resultado do teste FSLSM e do metadado descrito no objeto de aprendizagem do curso no início; um módulo de agente sensor, que captura o comportamento do aprendiz durante o processo para reagir as mudanças de forma automática e salva traços e características dos serviços mobiles de cada instancia no processo de aprendizagem.

Além disso, são implementados um agente de contexto, responsável pelo controle do processo de aprendizagem comparando com o caminho de aprendizagem inicial pelo agente guia com a observação do comportamento em cada instante e pela tomada de decisão; um agente grupo responsável por agrupar e identificar cluster próximos; um agente vizinho que cria matrizes de similaridade por um algoritmo denominado KNN; um agente controle que escuta as novas mudanças e envia para os demais; um agente de adaptação, que valida as experiencias; um agente revisão, que elabora uma nova situação de aprendizagem e atualizam a nova lista do conjunto próximo de estudante mudando a tabela de similaridade; um agente aprendizado, que apresenta a situação atual 
de aprendizagem e a futura para ser salva no banco de dados, e o agente coordenador, que coordena e sincroniza as mensagens dos agentes, gerencia a base com perfis, caminhos de aprendizagem, entre outras informações. Para a composição dos módulos foi utilizada a utilizada a plataforma JADE ${ }^{7}$ e o componente WSIG (Web Service Integration Gateway) como uma "porta de entrada-gateway" que oferece uma descoberta bidirecional e uma invocação remota ${ }^{8}$ de serviços web's para agentes desenvolvidos na linguagem.

O trabalho de Moro (2019) apresenta um chatbot para um Sistema Tutor Inteligente que possuí uma arquitetura baseada no trabalho de Pozzebon (2008). O chatbot visava atender as necessidades dos professores em conhecer sobre a ferramenta em questão atuando como capacitador dos usuários de diversas áreas de ensino. A arquitetura do STI possuí módulos de aluno, coordenador, professor, interface, domínio e pedagógico ao qual agentes inteligentes atuam em cada módulo. A atribuição do módulo professor e pedagógico com mais protagonismo é o grande diferencial do sistema e o aproxima de um ambiente virtual de aprendizagem inteligente. Além de disponibilizar o conteúdo o professor consegue definir de que forma apresenta determinado conteúdo de acordo com as características de comportamento e avaliação apresentadas no material para cada aluno, seja de uma sala, de um curso ou apenas utilizador.

A atribuição desta arquitetura é possível devido a sua composição por programação e algoritmos inteligentes, que se assemelha em muitos aspectos a sistemas como o Socrative ${ }^{9}$ o Duolingo ${ }^{10}$. Devido a esse aspecto de protagonismo do professor, se tornou essencial a atribuição do chatbot de apoio ao professor sobre o sistema, o chatbot proposto por Moro (2019) foi construído com a atribuição de um documento em formato JSON (formato de arquivo para trocas de dados) para composição da base de conhecimento, um classificador de palavras para processamento da linguagem natural e entendimento das dúvidas do professor e uma rede neural responsável pelo processo cognitivo do bot. Essa estrutura foi pensada depois de uma ampla pesquisa literária e em sistemas funcionais de empresas como IBM, GOOGLE e AMAZON, que utilizam estruturas semelhantes para criar modelos cognitivos funcionais e naturais aos bot's.

Na próxima seção será apresentada uma proposta de modelo de atribuição de agentes em um ambiente e-learning a partir das arquiteturas apresentadas e da atribuição de agentes conversacionais responsáveis para tentar minimizar os problemas apresentados na seção 2 .

\section{PROPOSTAS DE ARQUITETURA PARA MINIMIZAR OS PROBLEMAS CITADOS}

A arquitetura proposta se baseia no modelo clássico dos Sistemas Tutores Inteligentes (STI's) e na arquitetura proposta por Palomino (2013), Ghouch, EM-Naimi e Kouissi (2015) e Moro (2019) para uma possível aplicação em um AVA como o Moodle em uma disciplina da área de Matemática na Engenharia, com o uso de dois agentes conversacionais.

São acoplados ao sistema, agentes inteligentes formados por regras SE=>ENTÃO de programação que representam os seguintes módulos: um módulo aluno responsável por guardar informações estáticas e dinâmicas (como comportamento, desempenho, preferências.); um módulo professortutor responsável por armazenar informações do professor e por fornecer pareceres referentes a aprendizagem. Há também a necessidade de um modulo de domínio responsável por acompanhar os materiais alocados; um módulo interface que oferece a experiência visual tanto para aluno como para professor; e um módulo cognitivo responsável por fazer as intermediações de tutoria de forma mais 
próxima a natural e determinada determina o tipo apropriado de interface para cada usuário baseado em hardware e/ou software.

O módulo do aluno deve possuir: agente estático, agente coletor, agente atualizador e agente cognitivo do módulo do aluno. O módulo do professor possuí: agente estático, agente apoio, agente atualizador e agente cognitivo do professor. O domínio possui um agente estático, um agente dinâmico, um agente organizador e um agente cognitivo de domínio. O módulo de interface tem um agente de controle e um agente gatilho.

O modulo cognitivo atuará nos processos de entrega das informações e gerenciamento das informações do agente conversacional do aluno e do agente conversacional do professor, bem como nos processos de manipulação das variáveis de entrada, buscando as informações de todos os módulos para disponibilizar na interação. Ele atua diretamente também com o módulo de domínio para modificar o processo de disponibilidade ou sugestão de conteúdo.

No módulo do aluno, o agente coletor, coleta os traços de aprendizagem através de algoritmos preditivos de learning analítics ${ }^{11}$ obtidos dos últimos acessos do estudante no ambiente e envia os dados coletados ao agente atualizador que vai armazenar e decidir quais informações vai enviar e para qual módulo. Se há atualizações referentes a dificuldades ou acertos ele enviará então essas informações para o agente cognitivo do módulo do aluno e para o agente cognitivo de apoio do modulo professor.

O agente cognitivo do módulo do aluno será responsável por se comunicar e acionar o agente conversacional do aluno no módulo cognitivo para interagir com ele e caso a mensagem recebida seja de alguma dificuldade específica, material novo, ou acompanhamento o agente conversacional vai: a) explicar para o aluno que notou dificuldades a respeito de determinada atividade; b) sugerir materiais (que estão no módulo de domínio a respeito do tema que instigam a reflexão e o senso crítico); c) solicitar que o aluno resolva determinada questão (obtida de interação comparativa com o módulo de domínio) com seu apoio e interagir com ele a respeito de suas dúvidas com perguntas típicas de motivação e tutoria "Porque você acha isso? O que você espera disto? E se houver atribuição de reflexão providas pelo professor sobre determinado tema, sugerir de forma específica. d) perguntar se há alguma dúvida específica sobre o conteúdo apresentado e se não souber enviar essa dúvida ao agente cognitivo do professor; f) motivar e sugerir o aluno a encontrar mais materiais a respeito do assunto ou referentes a base pré-requisitos e/ou avisá-lo de possíveis atualizações de nota, material); e g) sugerir que ele procure a ajuda de um colega que tenha o nível de atualização para aquele domínio classificado como suficiente. Fora isso o agente conversacional pode atuar apenas respondendo a perguntas típicas, dessa forma toda informação que entra é armazenada no banco de dados para treinamentos futuros.

O agente cognitivo do módulo do professor será responsável por acionar o agente conversacional do professor para interagir com ele e caso haja alguma mensagem nova sobre dificuldade de determinado aluno relacionado a determinado tema vai: a) solicitar novos materiais para o módulo de domínio; b) solicitar alguma atividade de reflexão para sugerir para o aluno; c) se houver alguma nova atualização no domínio a respeito de avaliação entregue, avisar o professor que há uma nova entrega; d) sugerir modelos metodológicos de aplicação do seu conteúdo de acordo com as atualizações de dificuldade e aprendizagem do aluno. Essas informações a respeito de domínio são realizadas entre o agente cognitivo do módulo do professor, os agentes de interface, e o agente cognitivo de domínio. 
Tanto o agente conversacional do aluno como o agente conversacional do professor salvam as informações referentes a entradas do usuário no banco de dados, que servirão de apoio na melhoria do agente. Os agentes são treinados a partir das interações por uma base de dados JSON e de regras geradas pelos agentes, uma rede neural do tipo LSTM (arquitetura de rede neural do tipo recorrente com memória longa) treinada com as informações referentes aos processos ocorridos (que vai possuir uma memória ideal para aprender com a experiencia de aprendizado) e um classificador de linguagem natural de máquina que entenda a linguagem natural conversar de forma natural e traduzir as informações provenientes das regras para o estudante e com o professor.

\section{DISCUSSÃO}

Com Base em Aldowah, Al-Samarraie e Fauzy (2019), o tratamento dos dados relativos ao traço de aprendizagem obtido por learning analítics é direcionado aos agentes cognitivos de acordo com o uso e necessidade para: (a) Geração de estatística que serão usadas em avaliação formativa; (b) Diagnóstico de aprendizagem; (c) Estado de ânimo e motivação e interação entre agente conversacional e aluno promovendo maior proximidades visando diminuir a evasão do aluno no curso e-learning.

Esse direcionamento permite proporcionar a cada agente atuante no sistema dados relativos ao aluno, que possam auxiliar no processo de retenção e entendimento de problemas matemáticos, de cálculo por exemplo. Um módulo pode sugerir materiais de acordo com o nível de dificuldade do aluno e com suas falhas relacionadas a falta de requisitos. Além disso, o direcionamento permite ao professor entender quais tipos de materiais complementares deve alocar na ferramenta e em que ponto o aluno possuí fraquezas, para tentar evitar possíveis evasões provenientes do não entendimento dos problemas, da falta de relação com outras disciplinas, da falta de interação com o professor ou com outros alunos que entendem determinados problemas.

A atribuição de um agente conversacional que atue de forma inteligente tanto nos módulos do professor como no módulo do aluno, como um tutor, possibilita trazer a partir da interação natural esses problemas e possíveis soluções aos professores e aos alunos, atuando como um auxílio, de forma motivacional e mantendo o engajamento do aluno com o material atribuído. Ao tentar resolver um problema matemático, o aluno pode ter através das interações com o chatbot materiais de apoio, tarefas de reflexão, e entendimento sobre suas dificuldades e pontos a melhorar. Do mesmo modo, um chatbot para o professor pode auxiliá-lo a verificar metodologias que melhor se enquadrem ao processo de cada aluno, avaliações, e entendimento sobre quais pontos devem ser melhorados.

Donggil, Oh e Rice (2017) enfatizam que um agente conversacional pode atuar de forma pedagógica atuando como humano na interface e facilitando interações entre alunos e conteúdo, esse tipo de agente é especialista na entrega de conteúdo instrucional. O sistema pode realizar perguntas e entregar uma resposta ao aprendiz, o auxiliando a refletir de melhor em atividades realizadas a aprendizagem online.

\section{CONCLUSÃO}

Em disciplinas de matemática aplicadas a Engenharia como cálculo em ambientes e-learning, os problemas gerados são para muitos difíceis, requerem mais atenção do professor para com cada aluno e necessitam o cumprimento de pré-requisitos para uma melhor aprendizagem, evitando 
evasão e outros problemas apresentados neste trabalho. Existem diversos tipos de agentes inteligentes que podem atuar no aprendizado dos alunos em conjunto para construir uma aprendizagem personalizada e minimizar problemas comuns, esses agentes em conjunto formam uma rede multiagente, comuns em Sistemas Tutores Inteligentes, Ambientes Inteligentes de Aprendizagem, Ambientes Adaptativos e de instrução. A atribuição desses e outros agentes aos modelos tradicionais podem tornar esses sistemas massivos mais funcional e interessante tanto para os alunos quanto para os professores.

O objetivo deste artigo foi levantar os problemas relacionados ao quadro apresentado no parágrafo acima e possíveis soluções já disponibilizadas para que se pudesse apresentar uma proposta de arquitetura inteligente, baseada na literatura, composta por agentes inteligentes reativos e conversacionais.

Sugere-se para trabalhos futuros, a aplicação desta arquitetura utilizando o moodle ou outro sistema para verificar se ao utilizar métodos inteligentes de empresas como Google e IBM para personalização, motivação, adaptação e acompanhamento de aprendizagem ocorra menos evasão nas disciplinas de matemática nas engenharias. Estas aplicações estão sendo investigadas em projetos em andamento.

\section{REFERÊNCIAS}

AGUIAR, Eliane Vigneron Barreto; TAROUCO, Liane Margarida Rockenbach; REATEGUI, Eliseu.L. Supporting Problem-Solving in Mathematics with a Conversational Agent Capable of Representing Gifted Students' Knowledge. [online] Disponível em: https://ieeexplore.ieee.org/document/6758619. Acesso em: 05 out. 2020.

ALEXANDRU, Adriana; TÍRZIU, Eugenia; TUDORA, Eleonora; BICA, Ovidiu. Enhanced Education by Using Intelligent Agents in Multi-Agent Adaptive e-Learning Systems. Studies in Informatics and Control, Bucareste, v.24, n.1, p. 13-22, 2015.

ALDOWAH, Hanan; AL-SAMARRAIE, Hosam; FAUZY, Wan Mohamad. Educational data mining and learning analytics for 21st century higher education: A review and synthesis. Telematics and Informatics. Elsevier, v.37, p. 13-49, abr. de 2019.

AKUGIZIBWE, Edwin; AHN, Jeong Yong. Perspectives for effective integration of e-learning tools in university mathematics instruction for developing countries. Education and Information Technologies, Springer, v.25, n.5, p 889-903, ago. 2019.

CROWN, Stephen, FUENTES, Arturo, JONES, Robert, NAMBIAR, Rajiv, CROWN, Deborah. Anne G. neering: Interactive chatbot to engage and motivate engineering students. [online] Disponível em: https://peer.asee.org/ann-g-neering-interactive-chatbot-to-motivate-and-engage-engineering-

students. Acesso em: 19 de setembro de 2020.

DONGGIL, Song; OH, Eun Young; RICE, Marilyn. Interacting with a Conversational Agent System for Educational Purposes in Online Courses. [online] Disponível: https://ieeexplore.ieee.org/document/8005002. Acesso em:30 de set. 2020.

GHOUCH, Nihad El; EN-NAIMI, El Mokhtar; KOUISSI, Mohamed. Implementation of an Adaptive Learning System Based on Agents and Web Services. International Journal of Emerging Technologies in Learning, Vienna, v.15, n.14, p. 162 -178, 2020.

FERREIRA FILHO, Raymundo Carlos Machado. Estratégia de Elaboração de Projetos de Engenharia em Sistema Tutor Inteligente. Porto Alegre: UFRGS, 2008, Tese (Doutorado em 
Informática na Educação), Centro Interdisciplinar de Novas Tecnologias da Educação, Universidade Federal do Rio Grande do Sul, 2008.

FRIGO, Luciana Bolan. MathTutor: um ambiente interativo Multiagente para o ensino de Estrutura da Informação. Florianópolis: UFSC, 2002, Dissertação (Mestrado em Engenharia Elétrica), Centro Tecnológico, Universidade Federal de Santa Catarina, 2002.

FROZZA, Rejane; SILVA, Andréa Aparecida Konzen; SCHREIBER, Jacques Nelson Corleta; LUX, Beatriz; MOLZ, Kurt Werner; KIPPER, Liane Mahlmann; BORIN, Mirceia Pereira; CARVALHO, Amanda Brum; BAIERLE, Jorlei Luis; SAMPAIO, Lariane. Agentes Pedagógicos emocionais atuando em um Ambiente Virtual de Aprendizagem. RENOTE: Revista Novas Tecnologias na Educação, Porto Alegre, v. 9, n. 1, p. 1-10, jul. de 2011.

GHOUCH, Nihad El; EN-NAIMI, El Mokhtar; KOUISSI, Mohamed. Implementation of an Adaptive Learning System Based on Agents and Web Services. International Journal of Emerging Technologies in Learning, Vienna, v.15, n.14, p. 162 -178, 2020.

HASEGAWA, Dai; SAKUTA, Hirochi, UGURLO, Yusel. A Human-like Embodied Agent Learning Tour Guide for E-learning Systems. [online] Disponível em: 10.1109/EDUCON.2014.6826067. Acesso em: 10 out. 2020

HERPICH, Fabrício; NUNES, Felipe Becker; VOSS, Gleizer Bierhalz; JARDIM, Rafaela Ribeiro; MEDINA, Roseclea Duarte. Ambiente Virtual Imersivo para ensino em Redes de Computadores: uma proposta usando Agentes Inteligentes. [online] Disponível em: https://www.brie.org/pub/index.php/sbie/article/view/2930. Acesso em: 09 out. 2020.

IO H. N., LEE. C.B. Chatbots and conversational agents: A bibliometric analysis. [online] Disponível em: 10.1109/IEEM.2017.8289883. Acesso em: 20 out. 2020.

JACQUES, Patricia A; SEFFRIN, Henrique;RUBI, Geiseane; DE MORAIS, Felipe;GHILARDI,Cássio; BITTENCOURT, Gilbert; ISOTANI, Seiji. Rule-based expert systems to support step-by-step guidance in algebraic problem solving: The case of the tutor PAT2Math. Expert Systems with Applications, ScienceDirect, v.40, n. 14, p. 5456-5465, out. 2013.

KUYVEN, Neiva Larisane; ANTUNES, Carlos André; VANZIN, Vinicius João de Barros; SILVA, João Luis Tavares; KRASSMANN, Aliane Loureiro; TAROUCO, Liane Margarida Rockenbach. Chatbots na educação: Uma revisão sistemática da literatura. Revista Renote: Novas Tecnologias na Educação, v.16, n.1, p. 1-10, jul. de 2018.

LEONHARDT, Michele Denise. Um estudo sobre Chatterbots. Porto Alegre: UFRGS, 2005. Dissertação (Mestrado em Ciências da Computação), Instituto de Informática, Universidade Federal do Rio Grande do Sul, 2005.

LOCH, Birgit; LAMBORN, Julia. How to make mathematics relevant to first-year engineering students: perceptions of students on student-produced resources, International Journal of Mathematical Education in Science and Technology, Taylor \& Francis online, v.47, n.1, p. 29-44.

MORO, Francielli Freitas Moro. Protótipo de um chatbot para auxiliar o professor na utilização do Sistema Tutor Inteligente MAZK. Araranguá: UFSC, 2019. Dissertação (Mestrado em Tecnologias da Informação e Comunicação), Centro de Ciências, Tecnologias e Saúde, Universidade Federal de Santa Catarina, 2019.

PALOMINO, Cecília Estela Giuffra. Modelo de Sistema tutorial inteligente para ambientes virtuais baseado em agentes. Florianópolis: UFSC, 2013, Dissertação (Mestrado em Ciência da Computação), Centro Tecnológico, Universidade Federal de Santa Catarina, 2013. 
PASCHOAL, Leo Nathan; CHICON, Patricia Mariotto Mozzaquatro; FALCKENBACH, Gilse Antoninha Morgental. Concepção, Implementação e Avaliação de um Agente Conversacional com Suporte à Aprendizagem Ubíqua. Revista Renote: Novas Tecnologias na Educação, Porto Alegre, v. 15 n. 1, jul. de 2017.

RADOVIC, Slaviša; MARIC, Miroslav; PASSEY, Don. Technology enhancing mathematics learning behaviours: Shifting learning goals from "producing the right answer to " understanding how to address current and future mathematical challenges. Education and Information Technologies. Springer, v.24, n.4, p 103-126, jun. 2018.

RUSSEL, Stuart Jonathan; NORVIG, Peter. Inteligência Artificial: Uma abordagem Moderna. $2^{a}$ ed. Rio de Janeiro: Campus-Elsevier, 2004.

SANTOS, Valéria Cunha. Agentes inteligentes na educação a distância: uso de sistemas tutores inteligentes como auxiliares no estabelecimento da comunicação dialógica. In: Revista LinguaTec, Bento Gonçalves, v. 3, n. 6, p. 55-70, nov. 2018.

\section{AGRADECIMENTOS}

O presente trabalho foi realizado com apoio da Coordenação de Aperfeiçoamento de Pessoal de Nível Superior - Brasil (CAPES) - Código de Financiamento 001.

Submissão: 27/11/2020

Aceito: 05/01/2021

\section{Notas:}

${ }^{1}$ Modalidade de Ensino a Distância através de Tecnologias da Informação e Comunicação.

${ }^{2}$ Artificial Intelligence Markup Language: Linguagem derivada de XML, construída a partir de regras com o objetivo de facilitar a criação de ferramentas de computador para construção de diálogos semelhantes ao de humanos.

${ }^{3}$ Linguagem Natural de Máquina (NLP) e Redes Neurais Artificiais (RNA) são Técnicas de Inteligência Artificial para construção de sistemas inteligentes. O trabalho de Moro (2019) explica melhor as duas técnicas aplicadas a um agente conversacional.

${ }^{4}$ Aprendizagem Baseada em Problema: é uma metodologia que consiste, sucintamente, em: apresentar um problema para um grupo de alunos, que vai se reunir e discutir a procura de uma solução ideal, com o apoio de um tutor.

${ }^{5}$ Serial ATA: dispositivo para transferir dados em série de um computador para unidades externas.

6 JavaScript Object Notation: linguagem de programação para representação de dados Disponível em:<https://www.json.org>.

7 Java Agent Development Framework: Plataforma para desenvolvimento de agentes inteligentes. Disponível em: < https://jade.tilab.com/>

${ }^{8}$ Remote Method Invocation: Aplicação para chamadas remotas entre os objetos para aplicações desenvolvidas em JAVA.

${ }^{9}$ Sistema Inteligente para Aprendizagem. Disponível em: <https://www.socrative.com/>.

${ }^{10}$ Ambiente inteligente para ensino de idiomas online. Disponível em: <https://pt.duolingo.com/>.

11 Utilização de dados proveniente de estudantes em sistemas para análise e previsões em relação a sua situação em determinado curso. Isso é possível, a partir de algoritmos e levantamentos estatísticos. 